\title{
Berte aus grans piés von Adenet le roi und der berliner Prosaroman.
}

Das altfranz. Epos von Karls des Grofsen Mutter Berta lief im 13. Jahrh. in verschiedenen Versionen um. Die jüngste und beliebteste Fassung liegt uns vor in der um 1275 entstandenen Bearbeitung des brabanter Menestrels Adenet le roi (ed. A. Scheler, Bruxelles 1874) und in einem Prosaroman des 15. Jahrh. im Besitze der $\mathrm{kgl}$. Bibliothek zu Berlin (Ms. Gall. Fol. I30). In seinem Aufsatze zur Kritik der Bertasage (Ausg. u. Abhandl. 59. Marburg 1886 p. 5-10) hat A. Feist nachzuweisen gesucht, dals der Prosaroman (B), dessen nahe Verwandtschaft mit Adenets Gedicht (A) keinem Zweifel unterliegt, nicht aus letzterem geflossen ist, sondern dafs beide einer gemeinsamen Quelle entstammen.

Die Gründe, auf welche A. Feist seine Annahme stützt, sind folgende: Im Wald wird Berta von einem wilden Tiere überrascht, Adenet nennt es ausdrücklich eine Bärin (v. 1148. 1 154. 1161), der Prosaroman sagt: une grant beste sauvaige terrible et moult hideuse a merveilles, ne nomme pas l'istoire quelle estoit, mais bien dit qu'elle avoit la gueulle ouverte grande et orrible, etc. Ferner schildert uns Adenet v. 3219 ss. das Wappen, das der König dem Retter Bertas verlieh, und daran schliefst sich (v. 3226): „Depuis l'a li lignage porté et portera, Encor le porle cil qui l'erilage en a", eine offenbare Anspielung auf eine lebende, bekannte und durch die Beschreibung ihres Wappens genügend definierte Persönlichkeit, die Symon als ihren Stammvater betrachte. In der Prosa fehltdie Beschreibung des Wappens, statt dessen erhält Symon, als er zum Ritter geschlagen wird, den Beinamen „le Roy". Gestützt auf die Identität seines Beinamens mit dem des Symon, meint nun Feist, leitete Adenet le Roi in schelmischer Weise sich und sein Geschlecht von Symon ab; die Anspielung, die er v. 3226 macht, bezieht sich auf Adenet selbst, und das Wappen, das er beschreibt, ist sein eigenes. Die Hypothese ist geistreich, ihre Beweiskraft ist aber nicht durchschlagend. Denn erstens ist es blofs eine Vermutung, dafs Adenet sich selbst als Nachkomme Symons hat ausgeben wollen, er spielt nie wieder darauf an. Ist aber die Stelle wirklich in dem Sinne zu verstehen - was ja möglich ist -, so mag wohl auch der Prosaredaktor sie in dem Sinne verstanden haben, und er kamn alsdann leicht auf 
den witzigen Einfall gekommen sein, dem angeblichen Ahnherrn Adenets dessen Beinnamen le roi anzudichten; umgekehrt hege ich Bedenken anzunehmen, dafs Adenet seinen Beinamen als roi des menestrels mit dem Beinamen le Roy, den Symon vom König erhält, in Parallele gestellt hätte. Dafs B die in A vorgefundene Wappenschilderung ausliefs, dünkt mich nicht befremdlich: es ist etwas anderes um die Beschreibung eines Wappens und die eines Festes z. B. Was nun den Bären betrifft, wie leicht konnte der Verfasser des Prosaromans die genauere Angabe Adenets zuerst überseben haben, und schliefslich seine Schilderung eines nicht genannten, aber grausam wilden Tieres für packender gehalten haben. Immerhin sind die angeführten Beweisgründe nicht so überzeugend, dafs der Versuch eines Gegenbeweises von vornherein verzweifelt wäre.

Die Entscheidung, ob Adenet die Quelle des Prosaromans ist, oder ob beide eine gemeinsame Vorlage benutzt haben, ist nämlich von gröfserer Tragweite, als es scheinen könnte; es steht die litterargeschichtliche Bedeutung Adenets in Frage. Berte aus grans piés gilt nicht ohne Ursache als ein Meisterwerk der Verfallzeit der franz. Epik überhaupt und insbesondere als das Meisterwerk unseres Dichters, dessen hauptsächlichste Leistungen eben in derartigen Bearbeitungen älterer Epen bestehen. Bisher rühmte man an dem Roman nicht blofs die glatte Versifikation und den reinen, eleganten Stil, sondern vor allem die idyllische Einfachheit der Auffassung, die zarte Innigkeit der Gefühle, die geschickte Führung der in ihren Schilderungen freilich etwas breiten Erzählung; ganz besonders galt als Adenets Verdienst die glückliche Einführung Pipins durch den Kampf mit dem Löwen und die neue Wendung der Lösung, die ihrer ursprünglichen Roheit entkleidet worden ist. Das alles aber, die ganze Abfolge der Ereignisse, ihre Motivierung und der Geist, der die Erzählung beseelt, findet sich in auffallender Ähnlichkeit im Prosaroman wieder, und hätte sich demgemäls schon im Archetypus vorgefunden, Adenet hätte den gebotenen Stoff nur stilistisch, nur sprachlich und metrisch überarbeitet. Das ist nicht Adenets Nanier.

Vergleichen wir z. B. seine Enfances Ogier mit ihrem mutmafslichen Vorbilde, der ersten Branche der Chevalerie Ogier von Raimbert de Paris, so merken wir, dafs er nicht blofs seine Vorlage von 3102 Verse auf 8229 erweitert hat, sondern dafs er der Erzählung ein ganz neues Gepräge gegeben hat. Die abgerissene Leidenschaftlichkeit in Raimberts Gedicht, der ungehemmte Ausbruch der Gemütswallungen, die Kraft und die Anschaulichkeit der Erzählung sind durchweg abgeschwächt, vertuscht und verwässert; der Gang der Ereignisse ist in den Hauptzügen der gleiche, es giebt aber keinen einzigen Vorfall, den Adenet nicht von Grund aus umgestaltet hätte; sogar die unbedeutendsten Nebenumstände sind ohne Not abgeändert worden. So durchgreifend ist die Überarbeitung, dafs im ganzen Gedichte kaum acht Tiraden einigermafsen in Parallele zu bringen sind, und auch in diesen sind gleiche 
Verse und gleiche Gedanken eine Seltenheit. 1 Wie unwahrscheinlich, dafs derselbe Dichter sich in einem späteren Werke mit so geringen Abänderungen begnügt hätte!

Ich will nun versuchen zu zeigen, dafs der berliner Prosaroman dem Gange Adenets Schritt für Schritt folgt, dafs die Abweichungen, die er zeigt, aus allgemeinen Gründen leicht begreif bar sind; ja es scheint mir möglich aus der Prosa stellenweise die regelmälsige Abfolge männlicher und weiblicher Tiraden, die sich Adenet zur Aufgabe gemacht hat, zu erkennen.

Die Prosaerzählung ist im allgemeinen kunstlos, methodisch und breit. Indem sie Adenets Verse auflöst - denn von dieser Voraussetzung gehe ich nunmehr aus - , sucht sie die geschilderten Vorgänge durch natürliche Details anschaulich zu machen und die genaue Abfolge und den inneren Zusammenhang der Ereignisse herzustellen, während sie die wertlosen Einzelheiten, die nur dem Reime ihre Erwähnung verdanken, und die müfsigen Wiederholungen, in denen Adenet seine stilistische Gewandheit entfaltet, übergeht. Gesprochen wird viel und der Redaktor, der gern moralisiert, behandelt die Reden ganz frei. So fliefst seine Erzählung weder spannend noch ermüdend in gemächlicher Gleichmälsigkeit und gesprächiger Breite fort.

In diesem Geiste schliefst sich $B$ eng an $A$ an. Auf die Finleitung A I komme ich später zu reden. $\$ 2.3$ hat B ziemlich breit ausgeführt. Für $\$ 4-7$ verlangte eine geordnete Prosaerzählung, dafs zunächst Margiste und die Ihrigen eingeführt würden, infolge dessen hat $\mathrm{B}$ manches umgestellt, gekürzt und zusammengezogen. Eine erste Lücke in B umfalst $\$ 8-11(v .325) .^{2}$ Von hier bis $\S 19$ folgt B genau, nur wird die Besprechung zwischen Margiste und Aliste $\S \mathrm{I} 2$ weiter ausgeführt, die mit Thibert $\S$ I 3 kürzer gegefafst; das Knebeln Bertas wird später erwähnt, § I 7 Schlufs statt $\S$ I5 SchluIs; Bertas Klagen $\S$ I 8 werden nur kurz erwähnt: elle plourait piteusement et se doulousoit a part elle. Die zweite Lücke erstreckt sich von $\S 19$ Schlurs- $\$ 37$; B scheint die Klagen Bertas im Walde stark gekürzt zu haben, vgl. B 2 I ,mauldissans fortune qui de si hault lieu l'avoit en si bas fait cheoir, tresbuchée et amenée" mit A v. 855 ss. Die Erzählung beginnt wieder mit $\$ 38$ (v. 940);

1 Im ganzen sind es kaum mehr als I I Verse oder Halbverse, cfr. Chev. 479-491 =Enf. 815-821 (4 Verse). Chev. 660 = Enf. 1080. Chev. $906=$ Enf. 1356. Chev. 1229 ss. = Enf. 1707 ss. (Asson. resp. Reim auf ier). Chev. 1413. $1428=$ Enf. 1986. 1993. Chev. $1729=$ Enf. 2668. Chev. 1837 $=$ Ent. 285 I. Chev. $2744=$ Enf. 3756 .

2 Die Berliner Hs. bestand aus 5 Heften, die drei ersten hatten wahrscheinlich je 6 Bogen, das letzte 8. Es fehlt in Heft I der mittlere Bogen, in Heft II der äulsere Bogen und das zweite Blatt, in Heft III vermutlich die vier ersten Blätter und das sechstletzte. So entstanden vier Lücken in B; nach der modernen Paginierung zwischen p. 10-1I vier Seiten, p. 20-21 vier Seiten, p. 38-39 zehn Seiten(?), p. 42-43 zwei Seiten (?); die letzte Lücke ist noch nicht beachtet worden, es könnte viclleicht ein Überspringen des $\mathbf{A b}$ schreibers vorliegen. 
in B schläft Berta schon, als die Räuber sie gegen Morgen überfallen, darum sind $\S 39-4$ I bis auf weniges, z. B. Gebet an S. Julien, unbenutzt gelassen worden; aus $\S+2$ entnahm B Bertas Gelübde und schrieb alsdann $\S 44-46$ aus; $\S 47-49$ hat B ganz umgestellt, weil Adenets Erzählung ganz ungeordnet ist. Mit §50 beginnt der genauere Anschlurs wieder; es fehlen im folgenden $\$ 54$, cine überflüssige Mahlzeit, $\$ 55$ eine mürsige Rekapitulation, $\$ 57$ eine nochmalige Aufforderung der Constance, Berta möchte bei ihmen bleiben $=\S 53 ; \$ 58$ ist von $\mathrm{B}$ um Heiratsprojekte vermehrt. Die dritte Lücke entspricht $\$ 59-70$ letzter Vers. Bei der nun folgenden Reise Blanchefleurs hat sich $B$ die wirkungsvolle Begegnung der Königin mit dem verarmten Bauer $\$ 72.73$ nicht zu Nutzen gemacht. Von $\$ 74$ an ist die Übereinstimmung wieder sehr grofs, mit einer Lücke $=\S 75$ Mitte -76 Anfang. $\$ 79$ ist nur Wiederholung der umstehenden Verse, daher ist die Verwendung in B zweifelhaft, $\S 83$ ist ganz inhaltlos und fehlt; $\S 90-93$, Verwünschungen gegen die Verräter sind von B nur kurz angedeutet: $S_{i}$ deve's savoir que moult $y$ eut de parolles dictes ce soir en plain souper lant de nobles homme's comme d'aultres gens, während er deren Klagen vermilst: si ne dit mie l'istoire quels regres ilz firent ne quelles furent leurs parolles. Die gerichtliche Verhandlung $\$ 94-99$ hat B freier bearbeitet, eigentümlicherweise stellt er die Anwendung der Folter in Abrede; die weiblichen Tiraden sind hier fast sämtlich inhaltlos. Dann stimmt B mit $\S$ I OO wieder genau; $\S$ IOI und IO2 bis v. 2450 sind ohne Bedeutung und von $\mathrm{B}$ übergangen. Im folgenden ist der Anschlufs wieder vollständig bis auf $\$$ I I I $=\$$ I I 2, v. 27 IO ff. und $\$ 113=\S 112$ Schlufs, mürsige oder unrichtig angebrachte Wiederholungen; unklar ist ob $\$$ I 16 einfach übergangen worden ist. Das Hochzeitsfest $\$ 127-129$ wird in B ziemlich selbständig geschildert, die Belohnung Symon \$ I 29 Schlufs - I 3 I sehr gekürzt, ebenso die Rückreise nach Paris $\$ 132-138$. Von da an bis zum Schlufs ( $\$ 144)$ hat B manches umgestellt, aber nur die bedeutungslose Tirade 140 übersprungen.

Wir sehen also, dafs der Verfasser von B ein Gedicht vor sich hatte, das alle die Tiraden enthielt, welche für die Erzählung von Belang sind; wäre also A nicht Quelle von B, so hätte Adenet nur nichtssagende Wiederholungsstrophen zugedichtet, und das lediglich und allein des grammatischen Reimes wegen. Wer sich überzeugen will, wie grofs trotz der ganz verschiedenen Erzählungsund Schreibweise die Übereinstimmung beider Versionen oft ist, der vergleiche z. B. A $\$ 50-58,7$ I -89 , 102-1 26 mit den entsprechenden Abschnitten in B p. 3I-38, 39-55, 57-99.

Gerade an diesen Stellen werden wir auch Spuren der von Adenet gebrauchten poetischen Form erkennen; freilich hat der Nachweis des einstigen Reimes seine Schwierigkeit: denn Adenet hat seine künstliche Reimweise nur auf Kosten des Inhalts durchführen können, bald ist infolge derselben die Darstellung so abgerissen und zusammenhanglos, dafs der Bearbeiter sie vollständig 
neuordnen mufste, bald sind zwischen längere inhaltsvolle Tiraden kurze nichtssagende eingestreut, welche von $B$ einfach übergangen wurden; und gerade da wo die Erzählung am gedrängtesten und reichhaltigsten wird, gerade da ist Adenet dem grammatischen Reime abtrünnig geworden. Natürlicherweise bieten auch die auffälligsten Reime die grölsten Schwierigkeiten, deren Überwindung oft den einzigen, in Prosa nicht zur Geltung kommenden Wert solcher Tiraden bildet; leicht sind auch die charakteristischen Reimworte seltsam oder früh veraltet, und aufserdem ist ja B bemüht den Reim zu tilgen.

Aus allen diesen Gründen liefse sich der Mangel an bestimmten Anklängen im Einzelnen entschuldigen; ich will aber diesen Nachweis nicht führen, sondern greife gleich die beweiskräftigen Stellen heraus. B 28,32 heifst es: Par ung lundi matin à bonne estraine au commancement de la sepmaine etc. In diesen Worten erkennen wir deutlich A $§ 49$ :

Ce fu par un lundi au chief de la sepmaine

Que Berte fu trouvée en la forest dou Maine,

Dièus

Li a à cel lundi envoié bonne estraine.

Die Benutzung von $\S 48$ lärst sich nicht nachweisen; $\$ 50$ und 5 I sind inhaltlich treu wiedergegeben, Spuren des Reimes sind nicht vorhanden; für $\$ 52$ sind sie nur spärlich und unbedeutend, z. B. lit p. 32, $32 \mathrm{~s}$., die Übereinstimmung des Inhalts macht aber Reime auf $-i t$ wahrscheinlich; unzweifelhaft ist aber die Ausnützung von $\S 53$.

Berte, ce dist Constance, ne soyez desconfite,

Vo marrastre vous a et batue et laidite,

Dieus l'en rendra encore, sachiez, tout son merite.

$\mathrm{Vgl}$. B 33,14 Berthe, se. dit Constance, ne soiés point desconfite ne marrie... et ne pensées plus... a vostre maratre... car Dieu huy en rendra son loyer et merite. - Die Beweismomente sind vielleicht nicht bedeutend, aber sehen wir weiter: A $\$ 84$ Blancheflour fu assise sous l'ente en un prael, ist wohl nachgewiesen durch B 50,5. Ld où la royne de Hougrie parla d... Margiste... estoit un beau preau; $\S 85$ ist noch deutlicher zu erkennen am seltenen Worte favelle. A v. 2079 Ainsi li fait la vieille entendant la favele. B 50,25 et tant feit par sa cauteleuse flaterie et flavelle etc. Die nächste Tirade ist in $B$ sehr erweitert, Reimanklänge sind nicht vorhanden; und doch kann die Vorlage von B von A nicht stark abgewichen haben. Mit äufserster Deutlichkeit macht sich hingegen $\$ 87$ kenntlich. Die Stelle lautet B 52,14: Si grant paour eut Aliste qu'elle ne sceut que dire, car tout le corps luy tranbloit de courrous et d'ire, ... si n'avoit pas cause d'estre joyeuse... mais comme femme sans sens ou en frenesie se tourne et revire en son lit... Belle fille, je suis au cueur si dolante que je ne vous voie... car c'est la chose que plus desire......je suis plus descoulourée et plus jaune que cire. Vgl. damit: 
Grant paour ot la serve, plus que ne vous puis dire, Trestout li cors li tramble, n'a pas talent de rire, Ensus de Blancheflour se trait toujours et vire. „Fille, dist Blancheflour, tous li cuers me descire De ce que ne vous voi, car forment le desire." - „Mere, ce dist la serve, je suefre tel martire Que j'en sui aussi janne devenue com cire ....

Und dürfte man aus diesen auffälligen Anklängen nicht geradezu schliefsen, dafs die Vorlage von B stellenweise schon die Abfolge grammat. Reime auswies: so war sie doch sicherlich ein reingereimtes Gedicht in Alexandrinern, das mit Adenets Gedicht eine auffallende Ähnlichkeit hatte; und wer bezweifelte alsdann noch, dals es kein anderes als dieses war?

Übrigens wäre es im höchsten Grade befremdlich, dafs in Beginn des 15. Jahrh. ein Franzose zur Darstellung der Bertasage eine andere Quelle benutzt hätte als Adenets Berte aus grans piés. Die ganze Frage, ob B aus A geflossen ist, wäre von vornherein in bejahendem Sinne entschieden, wäre nicht auf Grund unzureichender Vergleichung das Gegenteil behauptet worden. ${ }^{1}$ Der berliner Prosaroman verliert 'somit wieder die litterargeschichtliche Bedeutung, die ihm ungerechtfertigter Weise beigemessen worden war, und Adenet bleibt der glückliche und geschickte Bearbeiter der Bertasage, wenn anders die Gültigkeit meiner Beweisführung anerkannt wird.

Aufser Adenets Gedicht hat B wohl aus keiner besonderen Quelle geschöpft. Er zeigt sich aber auch sonst in der alten Heldensage bewandert, vor allem mufste er Girart de Roussillon wohl kennen, da seine Histoire de la reyne Berte et du roy Pepin Fortsetzung einer Geschichte Karl Martells oder Girarts ist, von der wir sonst keine Kenntnis haben. ${ }^{2}$ Ferner kennt $B$ die Lothringergeste genauer; aus dem Karlssagenkreise erwähnt er im Widerspruch mit A $\$ 144$, dafs Karl Rolands Vater war (p. IOr); aus mündlicher Überlieferung wird ihm bekannt gewesen sein, dafs Berta nur einen gröfseren Fuf́s hatte, und dals Karl auf einem Karren erzeugt wurde (p. 106). Endlich verwertet $B$ noch einige ungenaue Geschichtskenutnisse: 751 Pipins Krönungsjahr; Childerich, angeblich dessen älterer Bruder ${ }^{3}$; vielleicht gehört auch hierher, dafs er Pipin 16

1 Z. B. sagt Feist, dafs in B Berta in Mannskleidern in den Wald gebracht wird, es heifst aber ausdrücklich p. 20,24: au departir de Paris . . . lui avoient v'estu les robes d'Aliste. - Ferner wird wiederholt gesagt, dals Berta als Symons Nichte ausgegeben wurde u. s. f.

2 Pepin qui ja estoit empereur par le moyen de Guerrart de Roussillon comme ja l'a racompté l'istoire ou livre precedant. - . . ou livre precedant ... n'estoit la matiere disposée si non a traicter du roy Charles Martel, de Girard de $R$. et de la grant mortelle guerre qu'ilz menerent l'ung contre l'uultre.

3 Vgl. Chron. de S. Denis: En celle année meisme fu rois clamez ... et sacrez... en l'an de l'incarnation de N.S. 750 . Childeris qui rois estoit apelez, fu tonduz et mis en une abbaïe. 
bis 17 Jahre alt nennt, als er mit dem Löwen kämpft. Aus diesen Momenten baut sich die Einleitung von B auf, die von der in A abweicht.

Das Ergebnis der vorgenommenen Prüfung ist nunmehr folgendes: Adenet le roi hat die Bertasage frei überarbeitet; seine Vorlage ist uns nicht bekannt, sie mochte aber unter den uns bekannten Versionen die größste Ähnlichkeit mit der vom poitevinischen Turpin excerpierten Fassung gehabt haben. Adenets Gedicht, Berte aus grans piés, ist seinerseits benutzt worden für das franz. Mirakel des 15. Jahrh., für unseren Prosaroman, und schliefslich für das bruchstückweise erhaltene niederländische Gedicht. ${ }^{1}$ Auf eine Untersuchung der übrigen Versionen der Bertasage und ihres gegenseitigen Verhältnisses, will ich gegenwärtig nicht eingehen; sie würde sich nur lohnen, wenn man die verschiedenen Fassungen von Mainet zu gleicher Zeit prüfte, aber diese sind mir nicht zugänglich. Zum Schlufs danke ich der Königlichen Bibliothek für die Erlaubnis, die Handschrift aufserhalb Berlins zu benutzen.

' $\mathrm{Hg}$. von $\mathrm{Ch}$. Piot im Bulletin de l'acad. royale de Belgique, $2^{e}$ série, t. XL, p. I55 ff. Bruxelles 1875 . Die erhaltenen Fragmente entsprechen folgenden Tiraden von Adenet: p. $163 \mathrm{~s} .=\S 115 ;$ p. 165 n. $1.2=\S 118$, n. $3=\oint 122 ;$ p. $161 \mathrm{~s}$. $=\oint 124$.

Ph. Aug. Beckek. 